INVESTIGACIÓN ORIGINAL

\title{
ACRETISMO PLACENTARIO EN EL INSTITUTO MATERNO INFANTIL (IMI) 1994 - 1999
}

\author{
Alain Davis Sjogreen, M.D. *, Lilia María Sánchez, M.D.**, \\ Jorge Andrés Rubio Romero, M.D. *** \\ Recibido: enero 12/2002 - Revisado: junio 20/2002 - Aceptado: agosto 27/2002
}

\section{PLACENTAACRETA IN THE INSTITUTO}

\section{MATERNO-INFANTIL 1994 - 1999}

\section{RESUMEN}

El acretismo placentario consiste en la adherencia anormal de la placenta a una decidua defectuosa o al miometrio, conduciendo a una demora en el alumbramiento o a la retención placentaria, asociada además a una hemorragia postparto que puede requerir o no histerectomía. Se realizó un estudio retrospectivo descriptivo de los casos de acretismo placentario encontrados y confirmados en el departamento de patología de nuestra institución entre el $1^{\circ}$ de enero 1994 y el 31 de agosto de 1999. Se revisaron los antecedentes gineco-obstétricos, aspectos clínicos e histopatológicos y se correlacionaron con los de la literatura. Dada la alta incidencia de parto por cesárea y su fuerte asociación con placenta previa y acretismo placentario, el obstetra deberá utilizar todos los métodos disponibles para diagnosticar y tratar esta patología.

Palabras clave: Placenta accreta, placenta

* Especialista en Obstetricia y Ginecología - Universidad Nacional de Colombia - instituto Materno Infantil. Bogotá D.C.

* Profesora asistente - Departamento de Patología - Facultad de Medicina - Universidad Nacional de Colombia - Instituto Materno Infantil. Bogotá D.C.

*** Profesor asistente - Departamento de Obstetricia y Ginecología - Universidad Nacional de Colombia - Instituto Materno Infantil. Bogotá D.C. íncreta, placenta pércreta, histopatología, hemorragia post parto, histerectomía.

\section{SUMMARY}

Placenta accreta is an abnormally attached placenta to an inadequate decidua and miometrium, conducing to a delayed third stage of delivery or to a placental retention, in association with postpartum haemorrhage that will require or not a hysterectomy. A retrospective descriptive study was designed and reviewed the records of the Pathology Department in our institution searching the placenta accreta histological confirmed cases between January first 1994 and august 30 1999. We review the clinical, obstetrical and histopathological features of all patients with diagnosis and we made a comparison with the literature. The higher incidence of caesarean section delivery today is strongly associated with the greater frequency of placenta previa and placenta accreta. Obstetricians must use all available methods to achieve an accurate diagnosis and treatment of this condition.

Key words: placenta accreta, placenta increta, placenta percreta, histopathology, postpartum haemorrhage, hysterectomy. 


\section{INTRODUCCIÓN}

El acretismo placentario (AP) describe cualquier implantación placentaria en la que hay una adherencia anormal a la pared uterina como consecuencia de la ausencia parcial o total de la decidua y el desarrollo imperfecto de la capa fibrinoide (capa de Nitabuch). En la placenta ácreta las vellosidades placentarias se fijan superficialmente al miometrio, en la placenta íncreta las vellosidades invaden en profundidad el miometrio y en la placenta pércreta las vellosidades invaden todo el espesor del miometrio y perforan la serosa. Esta adherencia anormal puede ser total, parcial o focal. ${ }^{4}$

La implantación del blastocisto en el endometrio humano es intersticial, por lo que este es rodeado en su totalidad por decidua. La decidua es la mucosa endometrial transformada por el efecto hormonal progestacional y la decidua del polo inferior del blastocisto (basal) limita la extensión del trofoblasto al miometrio y por lo tanto previene el AP.

Usualmente, la placenta se separa del miometrio debido a la contracción del miometrio contra la placenta inmóvil. Esto ocurre en un plano irregular de células deciduales friables justamente en la periferia de la capa de Nitabuch. La falta de la decidua evita la separación normal de la placenta después del parto por lo que esta puede ser retenida parcialmente o en su totalidad. Las áreas adherentes tienden a sangrar; además permiten acumular fibrina y coágulos alrededor de los tejidos placentarios y con el tiempo puede favorecer la formación de "pólipos placentarios" que requieran remoción quirúrgica. ${ }^{6}$

El AP es más frecuente cuando la decidua es defectuosa, y esto se observa asociado a diferentes entidades clínicas que implican en su mayoría manipulación del endometrio ${ }^{1,5}$. Dentro de estas entidades se incluyen: antecedente de cesárea, placenta previa, incisiones uterinas anteriores, legrados uterinos y cauterización endometrial, multiparidad (mayor de 3), edad materna (mayor a 35 años), presencia de divertículos, implantación cornual, miomas submucosos, síndrome de Asherman (adherencias intrauterinas), malformaciones uterinas (septos) y cirugía de reimplantación tubárica en el fundo uterino. ${ }^{7}$

Con el aumento de la incidencia de AP el obstetra debe utilizar todos los métodos disponibles para el diagnóstico preciso de esta condición, dentro de estos se incluye la ecografía obstétrica. Los criterios ultrasonográficos para el diagnóstico son: ${ }^{4}$

1. Ausencia o adelgazamiento (menos de $1 \mathrm{~mm}$ ) de la zona endometrial normal hipoecogénica en el segmento uterino inferior. Este criterio es el más sensible para realizar una detección selectiva de AP.

2. La presencia de espacios lacunares vasculares dentro del parénquima placentario, "apariencia de queso suizo", es un factor de riesgo para AP, aun en ausencia de otro hallazgo ultrasonográfico sospechoso.

3. El adelgazamiento, la irregularidad, o la disrupción focal de la interfase hiperecogénica entre las paredes uterina y vesical.

4. Extensión del tejido placentario (con la misma ecogenicidad del parénquima placentario) más allá de la serosa.

La resonancia magnética nuclear (RMN) puede ser útil en la valoración del compromiso vesical. Tiene la ventaja de visualizar la invasión de las paredes laterales y posteriores del útero en aquellos casos de implantación posterior de la placenta. La RMN se debe utilizar cuando la ultrasonografía no puede descartar la presencia de $\mathrm{AP}^{8}$

La evaluación de la placenta con flujo Doppler Color para el diagnóstico de AP no ha demostrado tener mayor sensibilidad que la evaluación ultrasonográfica en modo B. Por esta razón se considera el ultrasonido en Modo B como una herramienta diagnóstica básica para la identificación del 
acretismo placentario. El Doppler Color se puede utilizar como una técnica complementaria.

La importancia clínica del AP radica en la morbilidad y, a veces, la mortalidad materna secundarias. El manejo clínico y quirúrgico del AP por tanto está encaminado a la prevención de la hemorragia intraoperatoria incontrolable ${ }^{4}$. En estos casos la mortalidad puede ser tan alta como del $10 \%{ }^{4}$. Otras complicaciones que se presentan en estas pacientes son coagulación intravascular diseminada (CID), reacciones transfusionales, aloinmunización, sobrecarga de líquidos y con menos frecuencia infección. En el 7\% de las pacientes con AP puede ser necesaria la reexploración por sangrado postoperatorio. ${ }^{4}$

La morbilidad materna operatoria incluye: histerectomía, trauma intestinal, trauma urológico (trauma ureteral y laceración de vejiga). Existe también riesgo elevado de eventos tromboembólicos y alta incidencia de sepsis y síndrome de dificultad respiratoria del adulto (SDRA) secundario. ${ }^{4}$

El diagnóstico definitivo de AP se realiza mediante el estudio anatomopatológico del útero, el cual permite evidenciar la interfase úteroplacentaria para así demostrar la ausencia de decidua y el contacto directo de las vellosidades placentarias con el miometrio. En el examen macroscópico inicial se puede ver la inserción de la placenta y el nivel de penetración. (Figura 1) En el examen microscópico se confirma la ausencia de decidua y la adhesión anormal de la placenta. (Figura 2) Sin embargo, en muchas ocasiones no es posible realizar este examen ya que al laboratorio de patología solo es enviado material de legrado y/o la placenta por lo que el diagnóstico es en muchas ocasiones presuntivo, a no ser que el espécimen incluya miometrio.

\section{MATERIALES Y MÉTODOS}

Se realizó un estudio descriptivo de serie de casos. Durante el periodo de tiempo estudiado se

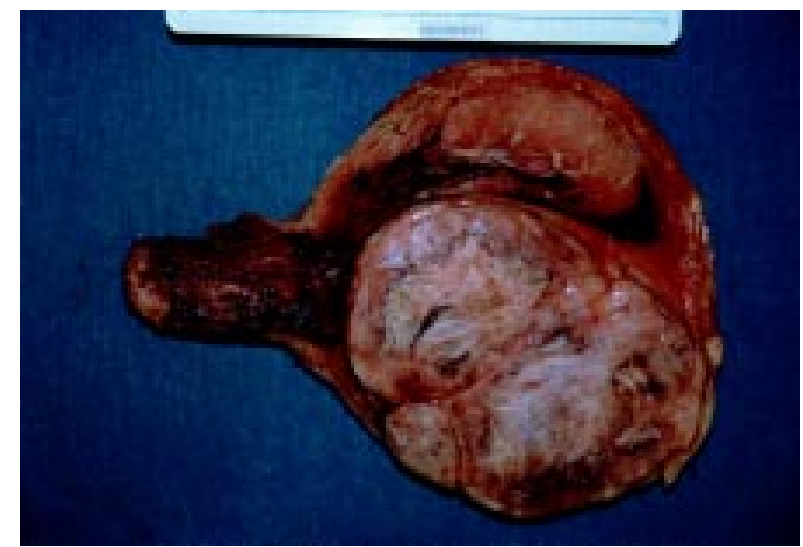

Figura 1. Aspecto al corte de un útero grávido en el que se evidencia la placenta adherida al miometrio al lado izquierdo de la cavidad endometrial. Del lado derecho se observa además un leiomioma intramural.

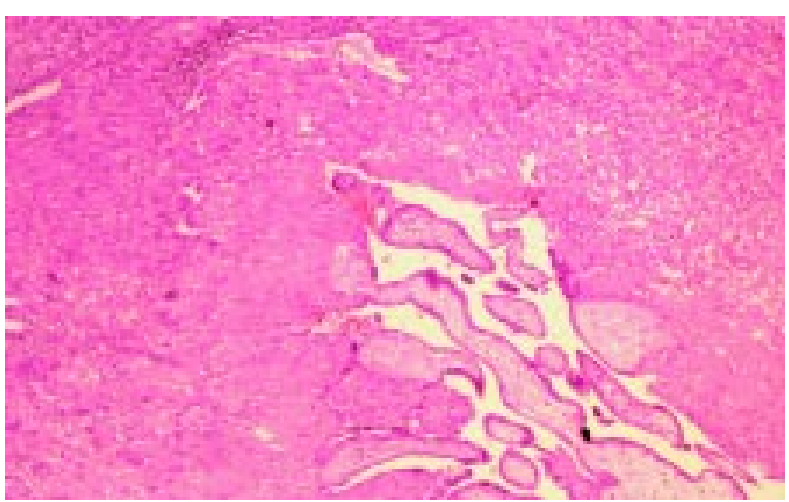

Figura 2. Interfase útero-placentaria con vellosidades coriales fibrosadas que invaden directamente el miometrio, en ausencia de decidua.

atendieron 30784 partos, se enviaron para estudio anatomopatológico 3781 placentas y 1866 úteros producto de cirugías ginecológicas y obstétricas.

Se revisaron los archivos del Laboratorio de Patología del Instituto Materno Infantil (IMI) en búsqueda de todos los especímenes con diagnóstico de AP al igual que el archivo de estadística y el Sistema Informático Perinatal (C.L.A.P) en el periodo comprendido entre el primero de enero de 1994 y el 31 de agosto de 1999 para seleccionar las pacientes que tuvieran el mismo diagnóstico. Se revisaron las historias clínicas y se llenó un formulario con datos como edad, paridad, gravidez, frecuencia de abortos, números de legrados, número de cesáreas, 
embarazos ectópicos, mortinatos, antecedente de cirugía uterina, localización anatómica de la placenta y de placenta previa, revisión uterina o cesárea, también el diagnóstico ecográfico de la localización placentaria, placenta previa, vía del parto, tratamiento quirúrgico del AP, así como las complicaciones del tratamiento del AP: 1 . necesidad de transfusiones, 2. mortalidad materna y perinatal, 3. resultado y complicaciones perinatales. Finalmente se describe también la correlación entre el estudio anatomopatológico y el diagnóstico clínico de AP.

Esta información se analizó en EpiInfo 6 y los resultados se exponen de manera descriptiva.

\section{RESULTADOS}

Al final de este estudio se encontraron 20 casos con diagnóstico de acretismo placentario, 16 de ellos a partir del estudio del útero y 4 productos de legrado obstétrico. Por cada 1.539 partos se encontró un AP, el cual correspondió a su vez a 1 de cada 282 placentas estudias en el laboratorio de patología.

El rango de edad encontrado en las pacientes fue de 20 a 37 años; la mayoría de las pacientes (65\%) se encontraba entre los 31 y 37 años. La edad gestacional osciló entre 28 y 40 semanas, hubo 3 pacientes en postparto inmediato sin registro de la edad gestacional. (Tabla 1).

La mayoría de los casos se encontró en pacientes con dos o más hijos (multíparas) y el antecedente de por lo menos un legrado previo (Tablas 2 y 3 ). El 50\% de las pacientes no tenía antecedente de cesárea previa (Tabla 4) y ninguna tenía antecedentes de cirugía uterina diferente a la cesárea.

El diagnóstico de la localización placentaria se hizo por ecografía, con la siguiente distribución: placenta previa $50 \%$ de las veces, fúndica (25\%), en la cara anterior y posterior (10\% para cada una) y canto izquierdo (5\%) (Figura 1). La mayoría de las placentas previas fueron centrales totales (45\%), seguidas de la central parcial (35\%), marginal (15\%) y de inserción baja (5\%).
Tabla 1. Edad gestacional de las pacientes con diagnóstico de acretismo placentario. IMI, 1994-1999.

\begin{tabular}{llcc}
$\begin{array}{l}\text { Edad } \\
\text { gestacional } \\
\text { (semanas) }\end{array}$ & $\mathrm{N}$ & $\%$ & $\%$ acumulado \\
\hline $28-31$ & 1 & 5 & 5 \\
\hline $32-35$ & 6 & 30 & 35 \\
\hline $36-39$ & 8 & 40 & 75 \\
\hline $40-43$ & 2 & 10 & 85 \\
\hline Sin dato & 3 & 15 & 100 \\
\hline \hline
\end{tabular}

Tabla 2. Antecedentes obstétricos de las pacientes con diagnóstico de acretismo placentario. IMI, 1994-1999.

\begin{tabular}{cccc} 
Gravidez & $\begin{array}{c}\text { Número } \\
\text { de pacientes }\end{array}$ & $\%$ & $\begin{array}{c}\% \\
\text { acumulado }\end{array}$ \\
\hline \hline 1 & 1 & 5 & 5 \\
\hline 2 & 4 & 20 & 25 \\
\hline 3 & 3 & 15 & 40 \\
\hline 4 & 3 & 15 & 55 \\
\hline 5 & 7 & 35 & 90 \\
\hline 7 & 1 & 5 & 95 \\
\hline 9 & 1 & 5 & 100 \\
\hline \hline
\end{tabular}

Tabla 3. Antecedente de legrado previo de las pacientes con diagnóstico de acretismo placentario. IMI, 1994-1999.

\begin{tabular}{cccc} 
Legrados & $\begin{array}{c}\text { Número } \\
\text { de pacientes }\end{array}$ & $\%$ & $\begin{array}{c}\% \\
\text { acumulado }\end{array}$ \\
\hline \hline 0 & 8 & 40 & 40 \\
\hline 1 & 10 & 50 & 90 \\
\hline 2 & 1 & 5 & 95 \\
\hline Sin dato & 1 & 5 & 100 \\
\hline
\end{tabular}

Tabla 4. Antecedente de cesárea de las pacientes con

diagnóstico de acretismo placentario. IMI 1994-1999.

\begin{tabular}{cccc}
$\begin{array}{c}\text { \# Cesáreas } \\
\text { previas }\end{array}$ & $\begin{array}{c}\text { Número } \\
\text { de pacientes }\end{array}$ & $\%$ & $\begin{array}{c}\% \\
\text { acumulado }\end{array}$ \\
\hline \hline 0 & 10 & 50 & 50 \\
\hline 1 & 6 & 30 & 80 \\
\hline 2 & 3 & 15 & 95 \\
\hline 3 & 1 & 5 & 100 \\
\hline \hline
\end{tabular}


La forma de nacimiento fue por cesárea segmentaría en 11 pacientes (55\%), vía vaginal en 7 pacientes (35\%) y cesárea corporal en 2 pacientes (10\%).

En 7 de las pacientes con antecedente de cesárea el AP se encontró sobre la histerorrafia y en 3 no hubo o no se pudo determinar la relación con la cicatriz.

El resultado perinatal fue bueno en 15 pacientes, adverso en 4 (con un fallecimiento) y en un caso el resultado fue desconocido. Describimos a continuación los diagnósticos finales de los neonatos con evolución adversa:

Caso 1: ano imperforado, fístula ano vestibular y sepsis neonatal.

Caso 2: asfixia perinatal severa, disfunción orgánica múltiple, sepsis y bronconeumonía.

Caso 3: asfixia perinatal severa, síndrome dismórfico, anormalidad cromosómica y prematurez.

Caso 4: enfermedad de membrana hialina, hemorragia pulmonar, hemorragia intracraneana, hidrocefalia y muerte neonatal.

En ninguna de estas 4 pacientes hubo sospecha clínica o ecográfica de A.P, al igual que en ninguna de las pacientes sometidas a cirugía y/o legrado. Una vez realizado el diagnóstico de AP el tratamiento que se realizó fue histerectomía abdominal total en 10 pacientes (50\%), histerectomía abdominal subtotal en 3 pacientes (15\%), histerectomía subtotal más ligadura de arterias hipogástricas en 1 paciente (5\%), histerectomía abdominal total más ligadura de arterias hipogástricas en 1 paciente (5\%), taponamiento uterino más histerectomía abdominal total más empaquetamiento pélvico en 1 paciente (5\%) y revisión uterina más legrado uterino en 4 pacientes (20\%).

En el 95\% de las pacientes se presentó hemorragia uterina postparto de las cuales el 90\% requirió transfusiones, se presentó también atonía

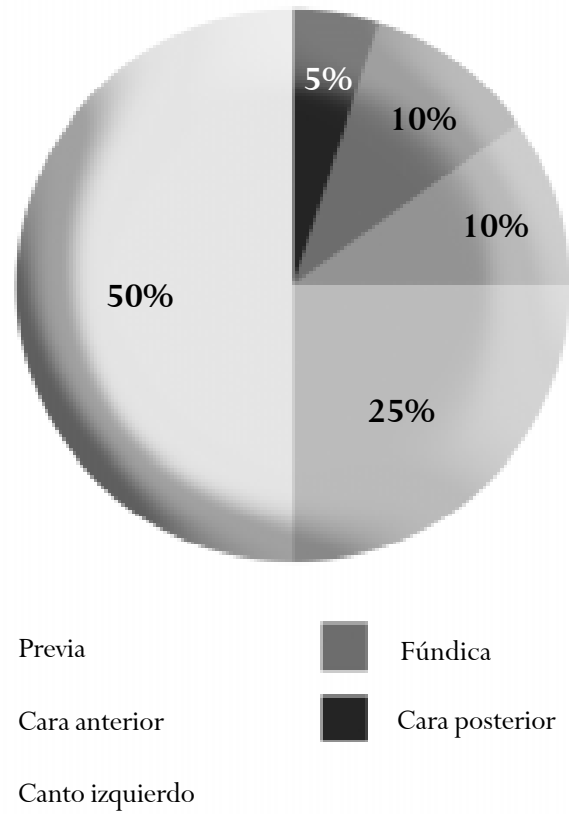

Figura 1. Diagnóstico ecográfico de la ubicación placentaria.

uterina en el 25\% de los casos, CID en el 15\%, sepsis puerperal en el 10\%, hematoma o absceso de cúpula vaginal en el 10\%, lesión vesical en el 15\%. Dos pacientes presentaron además complicaciones como hematoma de los paquetes infundíbulo-pélvicos y erupción cutánea, este último fue interpretado como una reacción post-transfusional (Figura 2). No se presentaron muertes maternas.

De las pacientes que recibieron transfusiones el 40\% recibió entre 3 y 5 unidades, el 31,6\% recibió 2 unidades (hasta un máximo de 19 unidades de glóbulos rojos transfundidos a una sola paciente). En el análisis de las historias observamos que el manejo de estas pacientes incluyó la transfusión de sangre durante la cirugía y en el postoperatorio, pero no se realizaron medidas tendientes a disminuir el sangrado transoperatorio como ligadura o embolización de arterias uterinas o hipogástricas. 
El diagnóstico anatomopatológico confirmó la presencia de AP en 12 de los 20 casos descritos. En los 8 casos restantes, el diagnóstico se basó en los hallazgos clínicos, el desenlace obstétrico y los hallazgos histopatológicos sugestivos vistos en los restos de placenta o el material de legrado recibido. De los casos confirmados con el estudio histopatológico del útero, ${ }^{12} 5$ pacientes tuvieron placenta pércreta $(42 \%), 4$ pacientes placenta ácreta (33\%) y 3 pacientes placenta íncreta (25\%). La inserción de la placenta fue previa en 3 de las 5 las pacientes con placenta pércreta.

\section{DISCUSION}

La incidencia de AP reportada en la literatura oscila entre 1 en 540 a 1 en 93000 partos, con una media de 1 en 7 000; en el periodo de tiempo estudiado en el IMI esta relación fue de 1 en 1 539 partos. $^{2}$

En la actualidad la alta frecuencia de parto por cesárea se asocia con mayor frecuencia a placenta previa, razón por la que se considera que ésta ha aumentado de 1 en 1000 embarazos en el año 1950 a 101 en 1000 en $1985 .^{3}$ El AP es más frecuente en pacientes con placenta previa o historia de cesárea anterior. ${ }^{1,2}$ El 25\% de las mujeres con antecedente de cesárea y placenta previa presentan AP de nuevo; el riesgo que aumenta al 50\% en las pacientes con historia de dos o más cesáreas y placenta previa. ${ }^{4}$
Llama la atención con relación al AP, que sólo 10 de las pacientes estudiadas tenían antecedente de cesárea, 6 de las cuales había sido sometida a una y 4 pacientes a más de una, lo cual resulta paradójico, pues es mayor la asociación esperada en mujeres embarazadas con este antecedente. En 7 de las pacientes con antecedente de cesárea se encontró relación entre la histerorrafia y el AP (70\%).

Se sabe que el $75 \%$ de las placentas pércretas se presentan en placentas de inserción previa o baja. En este estudio se realizó diagnóstico definitivo de AP en 12 de los 16 úteros estudiados, entre los cuales se encontraron 5 placentas pércretas (42\%) y 7 placentas pércretas y previas (58\%), lo cual muestra una proporción ligeramente menor a la descrita en la literatura. En este estudio se encontró placenta previa en 16 pacientes, central total en 9 (45\%), seguida de placenta previa central parcial en 7 (35\%).

Ya se ha mencionado que no se fue posible realizar el diagnóstico definitivo de AP en 8 pacientes debido a que se recibieron fragmentos de placenta 
o úteros en los cuales no se evidenió la interfase útero-placentaria. El diagnóstico final en estos casos fue presuntivo luego de correlacionar los datos de la historia clínica con el reporte de patología y el desenlace obstétrico. Muy probablemente estos fueron casos de placentas ácretas focales, parciales y totales cuya extracción manual o instrumentada fue exitosa.

Las complicaciones descritas en el presente estudio no difieren en forma significativa de las reportadas en la literatura. Además de las grandes pérdidas intraoperatorias de sangre que requirieron transfusiones masivas y de sus derivados, se evidenciaron complicaciones asociadas como coagulación intravascular diseminada (CID) y reacciones transfusionales. Se presentó infección sólo en el 10\% de los casos y fue necesaria la reintervención sólo en el caso de una paciente a quien se empaquetó la pelvis luego de la realización de la histerectomía abdominal; equivale al 5\%, porcentaje similar al reportado en la literatura. ${ }^{4}$

La morbilidad materna operatoria incluyó un porcentaje mayor de lesiones vesicales (15\%) comparado con el 2 a 3\% descrito en la literatura ${ }^{4}$. No se presentaron lesiones del intestino, eventos tromboembólicos, ni SDRA en ninguna de las pacientes.

La mortalidad descrita por la literatura es cercana al 10\%. Durante el periodo estudiado, no hubo desenlaces fatales, a pesar del estado crítico de las pacientes.

El AP ha aumentado su incidencia en las últimas décadas debido al aumento en la incidencia global de la operación cesárea y del número de cesáreas en cada paciente que las predispone a esta patología.

Cuando se consideran los aspectos demográficos de las pacientes a la luz de la obstetricia moderna, se espera enfrentar esta situación clínica con mayor frecuencia. Teniendo en cuenta los factores de riesgo y la disponibilidad de métodos diagnósticos eficaces para detectar el acretismo que permitan la preparación adecuada de la paciente, el obstetra tiene la posibilidad de manejar los casos que se presenten de manera planeada, con intervenciones multidisciplinarias, con el objetivo final de disminuir la morbilidad y la mortalidad maternas.

\section{CONCLUSIONES}

El AP es una entidad poco frecuente que se puede presentar durante toda la gestación y cuyo diagnóstico en la mayoría de los casos se realiza en el periodo posterior al parto por hemorragia uterina de magnitud variable, que puede llegar a ser difícil de controlar, con alta probabilidad de muerte para estas pacientes. Dado que existen factores de riesgo establecidos, es recomendable tener en mente esta entidad y realizar exámenes complementarios para su diagnóstico antes de finalizar la gestación, para prevenir o enfrentar exitosamente las complicaciones intraoperatorias más importantes que se puedan llegar a presentar.

\section{BIBLIOGRAFÍA}

1. Miller DA, Chollet JA, Goodwin TM. Clinical risk factors for placenta previa - placenta accreta. Am J Obstet Gynecol 1997:210-4.

2. O`Brien JM, Barton JR, Donaldson ES. The management of placenta percreta:conservative and operative strategies. Am J Obstet Gynecol 1996; 175:1632-8.

3. Clark S, Koonings P, Phelan J. Placenta previa/accreta and prior caesarean section. Obstet Gynecol 1985; 66:89-92.

4. Hudon L, Belfort MA, Broome DR. Diagnosis and management of placenta percreta: a review. Obstet and Gynecol Surv 1998; 53(8):509-17.

5. Cunningham F, Mac Donald PC. Williams Obstetrics. Fourth Edition. Masson 1996.

6. Benirscheke K, Haufmann P. Pathology of the human placenta. Third Edition, New York:SpringerVerlag:1995, p. 387-390. 
7. Lerwis S, Perrin E. Pathology of the Placenta. Second Edition, Churchill Livingstone, Philadelphia, USA 1999.

8. Thorp J, Councell B, Sandridge D, et al. Antepartum diagnosis of placenta previa percreta by magnetic resonance imaging. Obstet and Gynecol 1992; 80:506 $-506$.

9. Catanzarite V, Stanco L, Schimmers, et al. Managing placenta previa/accreta. Contemp Obstet Gynecol 1996; 41:66-95.
10.Alvarez M, Lockwood CJ, Ghidini A, et al. Prophylactic and emergent arterial catheterisation for elective embolization in obstetric haemorrhage. Am J Perinatol 1992; 9:441-444.

11.Dubois J, Gtarel L, Grignon A, et al. Placenta percreta: balloon occlusion and embolization of the internal iliac arteries to reduce intraoperative blood looses. Am J Obstet Gynecol 1997; 176:723-726.

12.AbdRabbo SA. Stepwise Uterine Devascularization: A novel Technique for Management of Uncontrolable Postpartum Haemorrhage with Preservation of the uterus. Am J Obstetric Gynecol 1994; 171:694-700. 